\title{
Motion Robust Magnetic Susceptibility and Field Inhomogeneity Estimation Using Regularized Image Restoration Techniques for fMRI
}

\author{
Desmond Teck Beng Yeo ${ }^{1,2}$, Jeffrey A. Fessler ${ }^{1,2}$, and Boklye Kim ${ }^{1}$ \\ ${ }^{1}$ Department of Radiology, University of Michigan Medical School, MI 48109, USA \\ \{tbyeo, fessler, boklyek\} @umich.edu \\ ${ }^{2}$ Department of Electrical Engineering and Computer Science, University of Michigan, \\ MI 48109, USA
}

\begin{abstract}
In functional MRI, head motion may cause dynamic nonlinear fieldinhomogeneity changes, especially with large out-of-plane rotations. This may lead to dynamic geometric distortion or blurring in the time series, which may reduce activation detection accuracy. The use of image registration to estimate dynamic field inhomogeneity maps from a static field map is not sufficient in the presence of such rotations. This paper introduces a retrospective approach to estimate magnetic susceptibility induced field maps of an object in motion, given a static susceptibility induced field map and the associated object motion parameters. It estimates a susceptibility map from a static field map using regularized image restoration techniques, and applies rigid body motion to the former. The dynamic field map is then computed using susceptibility voxel convolution. The method addresses field map changes due to out-of-plane rotations during time series acquisition and does not involve real time field map acquisitions.
\end{abstract}

Keywords: field inhomogeneity; susceptibility; geometric distortion; field map.

\section{Introduction}

In functional MRI (fMRI), time series images are acquired with high speed pulse sequences that are typically adversely affected by magnetic field-inhomogeneities. As a result, these images may be geometrically distorted or blurred, depending on the pulse sequence used. A static field-inhomogeneity map may be measured before or after a fMRI session to correct for such distortions [1,2], but it does not account for magnetic field changes due to head motion during the time series acquisition. To address this, several prospective dynamic field mapping techniques have been proposed $[3,4]$. However, they require pulse sequence modifications or high computational cost. Our work focuses on regularized image restoration methods to approximate dynamic field maps retrospectively without pulse sequence modifications.

A previous retrospective approach to approximate a dynamic field map applies rigid body transformations to an observed static field map [5], which may be sufficient only in the absence of significant out-of-plane rotations. In the presence of such rotations, that method may not be suitable since field-inhomogeneities may change nonlinearly [6]. Our approach is to retrospectively estimate the object's magnetic 
susceptibility $(\chi)$ map from an observed high-resolution susceptibility induced static field map using regularized image restoration principles. To compute the dynamic field maps, we apply rigid body motion to the $\chi$-map estimate, and apply 3D susceptibility voxel convolution (SVC) [7] to the resultant spatially translated/ rotated $\chi$-map. SVC is a deterministic, physics-based discrete convolution model for computing susceptibility induced field-inhomogeneity given a 3D $\chi$-map. Our main contributions include: (i) recognizing and formulating the inverse SVC problem for dynamic field map estimation, and, (ii) the implementation of a penalized least squares approach to solve the inverse problem. Another way to approximate the object's $\chi$-map is to segment a CT volume [6] into air, bone and soft tissue, and apply literature susceptibility values to different voxels. However, this may introduce segmentation errors and the use of incorrect susceptibility values. Our approach alleviates the burden of ensuring good accuracy in both the segmentation process, and the susceptibility values used. The approach is demonstrated with realistically simulated noisy 3D field maps of a spherical air pocket in water.

\section{Theory}

\subsection{Susceptibility Voxel Convolution for Field Map Computation}

Previous work [8] has shown that given an object with $K$ independent closed compartments of constant $\chi$ values, a Lorentz-corrected boundary element approach to computing the $z$-component of the $\chi$-induced magnetic field map, $\mathbf{B}_{\mathrm{p}}(\mathbf{r})$, yields

$$
\mathbf{B}_{\mathrm{p}}(\mathbf{r}) \approx \frac{\chi(\mathbf{r})}{3} \mathbf{B}_{0}+\frac{\hat{\mathbf{z}}}{4 \pi} \sum_{k=1}^{K}\left(\chi_{k}^{+}-\chi_{k}^{-}\right) \oint_{S_{k}} \frac{z-z^{\prime}}{|\mathbf{r}-\mathbf{r}|^{3}} \mathbf{B}_{0} \cdot \mathrm{d} \mathbf{S}^{\prime} \text { (Tesla), }
$$

where $\hat{\mathbf{z}}$ is a unit vector parallel to $\mathbf{B}_{0}, \chi_{k}{ }^{+}$and $\chi_{k}{ }^{-}$denote the susceptibilities outside and inside the $k^{\text {th }}$ compartment, respectively, $S_{k}$ is the $k^{\text {th }}$ surface, $\mathbf{r}^{\prime}$ is a surface point, $\mathrm{d} \mathbf{S}^{\prime}$ is perpendicular to the surface at $\mathbf{r}^{\prime}$. In the presence of out-of-plane rotations, the orientation of the surfaces with $\mathbf{B}_{0}$, i.e., $\mathbf{B}_{0} \cdot \mathrm{d} \mathbf{S}^{\prime}$, changes, thus resulting in nonlinear field map changes.

Susceptibility voxel convolution (SVC) [7] applies Eq. 1 directly to voxels of an object. Each voxel is defined as a closed six-sided compartment of uniform susceptibility. The dot product, $\mathbf{B}_{0} \cdot \mathrm{d} \mathbf{S}^{\prime}$, is non-zero only for the top and bottom surfaces of a voxel. Only the upper surface is used since the superposition principle allows each surface to be used only once in computing $\mathbf{B}_{\mathrm{p}}(\boldsymbol{x})$. The values of $\chi_{k}{ }^{-}$and $\chi_{k}{ }^{+}$are obtained from the $k^{\text {th }}$ voxel, and the voxel above it in the $z$ direction, respectively. The $\chi$-induced field equation now becomes

$$
\mathbf{B}_{\mathrm{p}}(\mathbf{r}) \approx \mathbf{B}_{0}\left[\frac{\chi(\mathbf{r})}{3}+\frac{1}{4 \pi} \sum_{k=1}^{K}\left(\chi_{k}^{+}-\chi_{k}^{-}\right) \int_{y_{k}-l_{y} / 2}^{y_{k}+l_{y} / 2} \int_{x_{k}-l_{x} / 2}^{x_{k}+l_{x} / 2} \frac{\left(z_{k}+l_{z} / 2-z\right)}{|\mathbf{r}-\mathbf{r}|^{3}} d x^{\prime} d y^{\prime}\right],
$$

where $\left(x_{k}, y_{k}, z_{k}\right)$ is the center of voxel $k$, and $l_{x}, l_{y}, l_{z}$ are the $x, y$, and $z$ lengths of a voxel. After discretisation in r, Eq. 2 becomes a 3D discrete convolution in space domain. The convolution kernel can be written as 


$$
\begin{aligned}
& K\left(x-x_{k}, y-y_{k}, z-z_{k}\right)= \\
& \left(z_{k}+l_{z} / 2-z\right) \int_{y_{k}-l_{y} / 2}^{y_{k}+l_{y} / 2} \int_{x_{k}-l_{x} / 2}^{x_{k}+l_{x} / 2}\left[\left(x^{\prime}-x\right)^{2}+\left(y^{\prime}-y\right)^{2}+\left(z_{k}+l_{z} / 2-z\right)^{2}\right]^{-3 / 2} d x^{\prime} d y^{\prime},
\end{aligned}
$$

and the SVC impulse response is

$$
d(h, i, j)=\frac{\delta\left(l-l^{\prime}\right) \delta\left(m-m^{\prime}\right) \delta\left(n-n^{\prime}\right)}{3}+\frac{[K(h, i, j-1)-K(h, i, j)]}{4 \pi},
$$

where $(l, m, n)$ denotes the voxel where $\mathbf{B}_{\mathrm{p}}$ is to be calculated, and $\left(l^{\prime}, m^{\prime}, n^{\prime}\right)$ denotes voxels in the field of view. The discrete convolution of Eq. 2 becomes

$$
\mathbf{B}_{\mathrm{p}}(l, m, n)=\mathbf{B}_{0} \sum_{l^{\prime}, m^{\prime}, n^{\prime} \in \mathrm{FOV}} \chi\left(l^{\prime}, m^{\prime}, n^{\prime}\right) d\left(l-l^{\prime}, m-m^{\prime}, n-n^{\prime}\right),
$$

which can be computed with

$$
\mathbf{B}_{\mathrm{p}}=\mathbf{B}_{0} \chi * * * d=\mathbf{B}_{0} \mathfrak{I}_{3 D}^{-1}\left(\mathfrak{I}_{3 D}(\chi) \mathfrak{I}_{3 D}(d)\right),
$$

where $* * *$ denotes $3 \mathrm{D}$ convolution, and $\mathfrak{I}_{3 D}$ denotes $3 \mathrm{D}$ Fourier transform. The discrete convolution can also be written in matrix-vector notation as

$$
\mathbf{B}_{\mathrm{p}}=\mathbf{D} \boldsymbol{\chi},
$$

where D denotes the SVC "system" matrix and $\chi$ is the column-stacked $\chi$-map vector. The $\chi$-induced field map in $\mathrm{Hz}$ is $\Delta \boldsymbol{\omega}_{\mathrm{p}}=\gamma \mathbf{B}_{\mathrm{p}}$, where $\gamma$ is the gyromagnetic ratio of hydrogen.

\subsection{Dynamic Field Map Estimation with Penalized Weighted Least Squares Estimation of Magnetic Susceptibility Map - A 3D Image Restoration Approach}

A static field map, $\Delta \boldsymbol{\omega}_{\text {static, }}$ is typically approximated by taking the phase difference of a pair of gradient-echo images acquired at two different echo times [9], and may be composed of susceptibility and non-susceptibility induced field inhomogeneity sources. The two complex-valued images may be denoted by

$$
\begin{gathered}
I_{j}^{\mathrm{TE} 1}=f_{j}+\varepsilon_{j}^{\mathrm{TE} 1}, \\
I_{j}^{\mathrm{TE} 2}=f_{j} e^{-i \Delta \omega_{\mathrm{static},} j \mathrm{TT} E}+\varepsilon_{j}^{\mathrm{TE} 2},
\end{gathered}
$$

where $f$ is the complex transverse magnetization of the object, $j$ is the voxel number, $\Delta \mathrm{TE}$ is the echo time difference, and $\varepsilon$ is independent identically distributed MR Gaussian noise. The echo time difference is typically small to prevent phase wrapping. In previous work [10], the maximum likelihood estimator for $\Delta \omega_{\text {static }}$ was shown to be

$$
\Delta \hat{\boldsymbol{\omega}}_{\text {static }}=\underset{\Delta \boldsymbol{\omega}_{\text {static }}}{\arg \min } \sum_{j}\left|I_{j}^{\mathrm{TE} 2} I_{j}^{\mathrm{TE} 1}\right|\left[1-\cos \left(\angle I_{j}^{\mathrm{TE} 2}-\angle I_{j}^{\mathrm{TE} 1}-\Delta \omega_{\text {static }, j} \Delta \mathrm{TE}\right)\right] .
$$


Ignoring phase wrapping, and decomposing $\Delta \omega_{\text {static }, j}$ into susceptibility and system induced parts, i.e., $\Delta \omega_{\text {static }, j}=\gamma[\mathbf{D} \chi]_{j}+\Delta \omega_{\text {sys }, j}$, and since a minimum exists when the cosine term in Eq. 10 is equal to one, the maximum likelihood estimator for $\chi$ is

$$
\hat{\chi}_{j}=\frac{1}{\gamma}\left[\mathbf{D}^{-1}\left[\frac{\angle \mathbf{I}^{\mathrm{TE} 2}-\angle \mathbf{I}^{\mathrm{TE} 1}}{\Delta \mathrm{T} E}-\Delta \boldsymbol{\omega}_{\mathrm{sys}}\right]\right]_{j} .
$$

For simplicity, we assume that $\Delta \boldsymbol{\omega}_{\text {sys }}$ is negligible, or can be measured empirically. Since the SVC frequency response has very small values at some frequencies, the inverse SVC problem is ill-posed, and thus 3D smoothness regularization is desirable when solving for $\chi$. We propose to use a quadratic penalized weighted least squares (QPWLS) image restoration approach to estimate $\chi$ by minimizing the cost function

$$
\Psi(\chi)=\frac{1}{2}\|\mathbf{g}-\gamma \mathbf{D} \chi\|_{\mathbf{W}}^{2}+\beta\|\mathbf{C} \chi\|^{2},
$$

where $\mathbf{g}$ is the observed static field map $\left(\angle \mathbf{I}^{\mathrm{TE} 2}-\angle \mathbf{I}^{\mathrm{TE} I}\right) / \Delta \mathrm{TE}, \mathbf{W}$ is a weighting matrix that assigns higher weights to voxels where MR image intensity, i.e., $\mid I_{j}^{\mathrm{TE} 2} I_{j}^{\mathrm{TE} 1} \mathrm{I}$, is higher, $\beta$ is a regularization parameter that determines the amount of smoothing, and $\mathbf{C}$ is a first order finite-differencing matrix. We minimize the cost function using the conjugate gradient algorithm. Any available motion estimates for each slice or volume in the fMRI time series can then be used to rotate or translate the $\chi$-map estimate. Since the SVC impulse response is linear shift invariant and depends only on the voxel size and orientation with respect to $\mathbf{B}_{0}$, it remains unchanged when a $\chi$-map undergoes rigid body transformation. Thus, the same SVC matrix used in estimating the $\chi$-map can be used to compute the dynamic field map after the desired motion has been applied.

The proposed QPWLS method was compared to three other methods of approximating the dynamic field map from an observed field map: thresholded inverse filtering [11], Wiener filtering [11], and direct rotation of the observed field map to the tilted positions [5]. The thresholded inverse filter ignores noise statistics and amplifies noise in frequency bands where the SVC frequency response has small values. To mitigate the latter, while preserving as much spatial information as possible, the threshold parameter needs to be chosen carefully, usually in an empirical manner. The Wiener filter assumes that $\chi$ and the additive field map noise are stationary processes, and assumes that their power spectra may be estimated accurately, which is often not true in the $\chi$ estimation problem.

\section{Methods}

\subsection{Data Simulation}

To measure the algorithms' performances, we generated 91 pairs of ground truth $\chi$-maps of a simulated, off-centered spherical air pocket $\left(\chi_{\text {air }}=0.04 \mathrm{ppm}\right.$ [7]) in water $\left(\chi_{\text {water }}=-9.05 \mathrm{ppm}[7]\right)$ that was rotated counterclockwise about the $x$-axis by angles from $0^{\circ}$ to $180^{\circ}$, in increments of $2^{\circ}$. The dataset with $0^{\circ}$ rotation was defined to be in the non-tilted position. In addition, an observed field map in the $0^{\circ}$ position was generated. Each $256 \times 256 \times 256$ dataset had a voxel size of $1 \mathrm{~mm} \times 1 \mathrm{~mm} \times 1 \mathrm{~mm}$. 
A SVC impulse response was formed (Eq. 4) and applied to all the ground truth $\chi$-maps (Eq. 6) with $\mathrm{B}_{0}=1.5 \mathrm{~T}$. The resultant ground truth field maps were then cropped to $128 \times 128 \times 128$ voxel volumes. To form the weighting matrix $\mathbf{W}$ in Eq. 12 , we simulated an image intensity map, $\mathbf{f}$, with zeros in the air pocket region (no MR signal), and 100 in the water region. With the non-tilted ground truth field map $\left(\Delta \boldsymbol{\omega}_{\text {static }}\right)$, an arbitrary value for $\Delta \mathrm{TE}$ (short enough to avoid phase wrapping), and $\mathbf{f}$, we used Eqs. 8 and 9 to generate two independent, complex Gaussian images, each with a SNR of 100.0. An observed non-tilted field map, g, shown in Fig. 1(a), was then computed as described in the Theory section.

\subsection{Experiments}

The main goal of this work is to accurately estimate rotated $\chi$-maps and field maps given (i) an originally observed non-tilted susceptibility induced field map, and, (ii) the respective rotation angles about the $x$-axis. We compared the field map estimation accuracy of our proposed method with those of thresholded inverse filtering, Wiener filtering and direct rotation of the original observed field map to tilted positions.

The first part of the experiment involved the estimation of the original, non-tilted $\chi$-map using the various methods. We applied the SVC matrix to these $\chi$-map estimates to compute field map estimates from which a few slices are shown in the top row of Fig. 1. Root mean-square-error (RMSE) values were then computed with reference to the $3 \mathrm{D}$ ground truth non-tilted field map, $\Delta \boldsymbol{\omega}_{\text {static }}$. In the second part of the experiment, the $\chi$-map estimates from the first part were all rotated about the $x$-axis by the same range of values used to create the 91 pairs of ground truth maps, i.e., $0^{\circ}$ to $180^{\circ}$ in increments of $2^{\circ}$. The SVC matrix was again applied to these rotated $\chi$-map estimates to compute the dynamic field map estimates. RMSE values were then computed with reference to the $3 \mathrm{D}$ ground truth tilted field maps. The second row in Fig. 1 shows a few field map slices of the object rotated by $45^{\circ}$, and the field map RMSE values for all positions and methods were plotted in Fig. 2.

The QPWLS implementation was built upon previous work [12], and 50 iterations of the algorithm were performed for each dataset with $\beta=0.7$. The initial guess for the conjugate gradient algorithm was a volume filled with zeros. For the thresholded inverse filter, a threshold value of $10(0.2 \%$ of the maximum absolute value of the inverse of the SVC frequency response) was used. A flat $\chi$ power spectrum was used for the Wiener filter to contrast its higher dependence on object prior information with that of the spatial smoothness prior in the proposed method. All algorithms were implemented in MATLAB (The Mathworks Inc., Natick, MA, USA) and C++, and executed on Intel Pentium 4 Xeon 3.0GHz CPUs.

\section{Results}

The RMSE values over entire 3D field map estimates for all rotated positions using the various field map estimation methods are shown in Fig. 2. Comparing all the methods, it was observed that the QPWLS method had the lowest (best performing) RMSE values, and RMSE variability, across all rotated positions. 


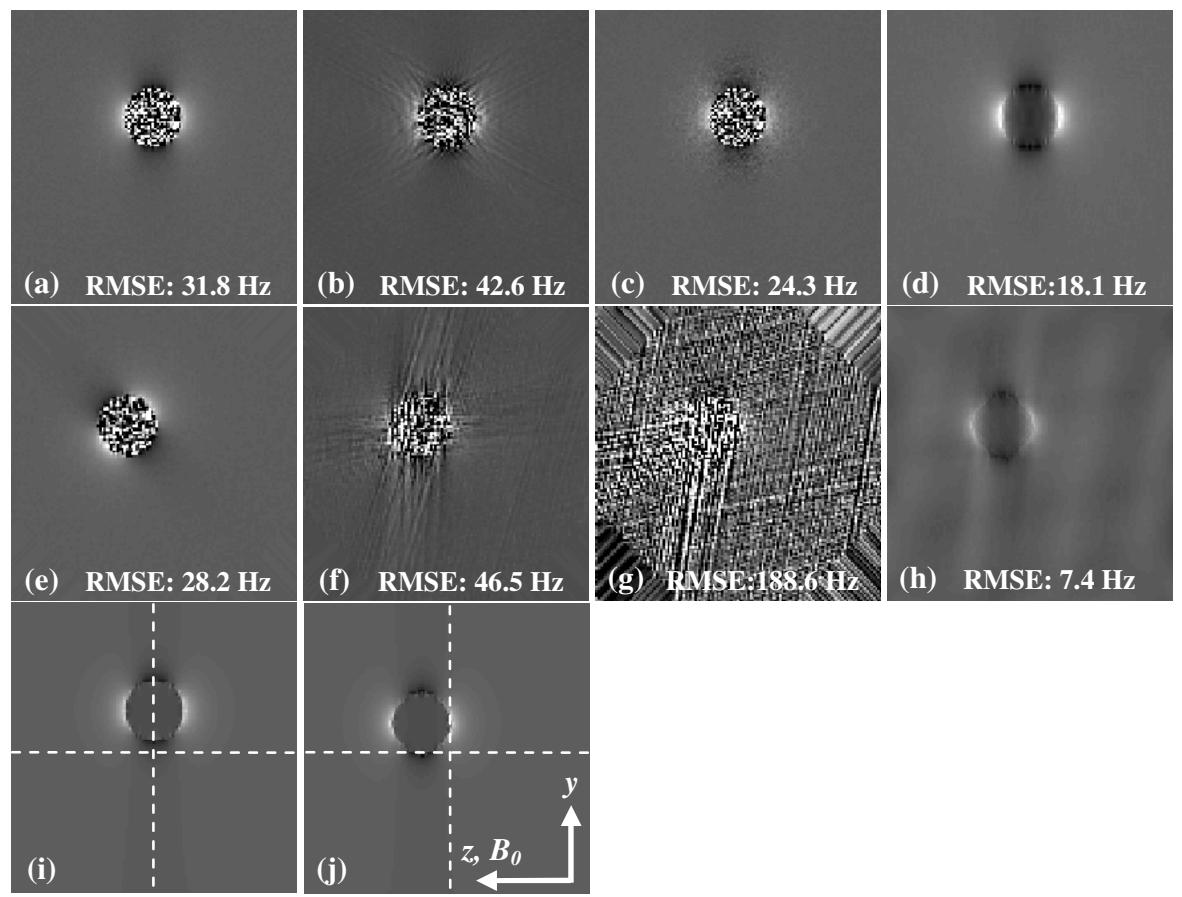

Fig. 1. (Top row) Non-tilted field map slice ( $y-z$ plane) from (a) originally observed field map, (b) thresholded inverse filter estimate, (c) Wiener filter estimate with flat object power spectrum, (d) QPWLS estimate with $\beta=0.7$. (Second row) $45^{\circ}$ rotated field map slice from (e) rotation of original observed field map, (f) application of SVC on rotated estimate of $\chi$ from thresholded inverse filter, $(\mathrm{g})$ application of SVC on rotated estimate of $\chi$ from Wiener filter, (h) application of SVC on rotated estimate of $\chi$ from QPWLS. (Bottom row) Ground truth field maps for (i) non-tilted, and (j) $45^{\circ}$ tilted positions. Display scale: -200 to $450 \mathrm{~Hz}$.

Fig. 1 shows the field map estimates' slices at the same spatial location in the $y-z$ plane when the object was in the $0^{\circ}$ and $45^{\circ}$ rotated positions, i.e., same slice position from two rotation angles in Fig. 2. The $x$-axis points into the plane of the page. It was observed that the field map estimates in the spherical air region were invariably noisy for the inverse filter and Wiener filter in Figs. 1(b), 1(f), and Figs. 1(c), 1(g), respectively. The noise in this region was greatly reduced in the QPWLS estimates in Figs. 1(d) and 1(h) because the weighting matrix suppressed the data fidelity requirement in the air region, which allows for smoother $\chi$-map estimates in this region. Since there were less abrupt susceptibility changes in the regularized $\chi$-map estimates, the resultant field inhomogeneity estimate in the air region was small and smooth. For an EPI pulse sequence with a typical phase encode pixel bandwidth of about $20 \mathrm{~Hz}$, the QPWLS RMSE values $(<20 \mathrm{~Hz})$ in Fig. 2 represent errors of less than one pixel shift. In contrast, the RMSE values for the other methods $(>20 \mathrm{~Hz})$ translate to errors of more than one pixel shift, which may reduce the accuracy of geometric distortion algorithms that depend on field maps. The SVC field map computation time was 1.5 
secs for a $128 \times 128 \times 128$ voxel $\chi$-map. The computation times for $\chi$-map estimation using the thresholded inverse filter, Wiener filter and QPWLS method were 4.1 secs, 5.8 secs, and 5.6 secs (one iteration), respectively.

\section{Discussion and Conclusions}

The proposed method estimates dynamic susceptibility induced field maps from an observed static susceptibility induced field map, while accounting for the proper MR noise model. It does not require segmentation or pulse sequence modifications, and may yield higher resolution dynamic field maps that address nonlinear changes due to out-of-plane rotations. Fig. 2 shows quantitatively that the QPWLS RMSE values were the lowest (best performing) among all the other methods. Figs. 1(d) and 1(h) show qualitatively that the field map estimates were close to the ground truths. For our spherical air pocket in water, nonlinear field map changes would typically be worst at the $90^{\circ}$ position, hence the peak in Fig. 2 for the method that simply rotates the observed field map. In contrast, the low QPWLS RMSE variability across rotation angles in Fig. 2 suggests that the method performs reasonably well regardless of rotation angles. Further improvements may be possible upon optimizing the choice for the regularization parameter, coupled with the implementation of regu-

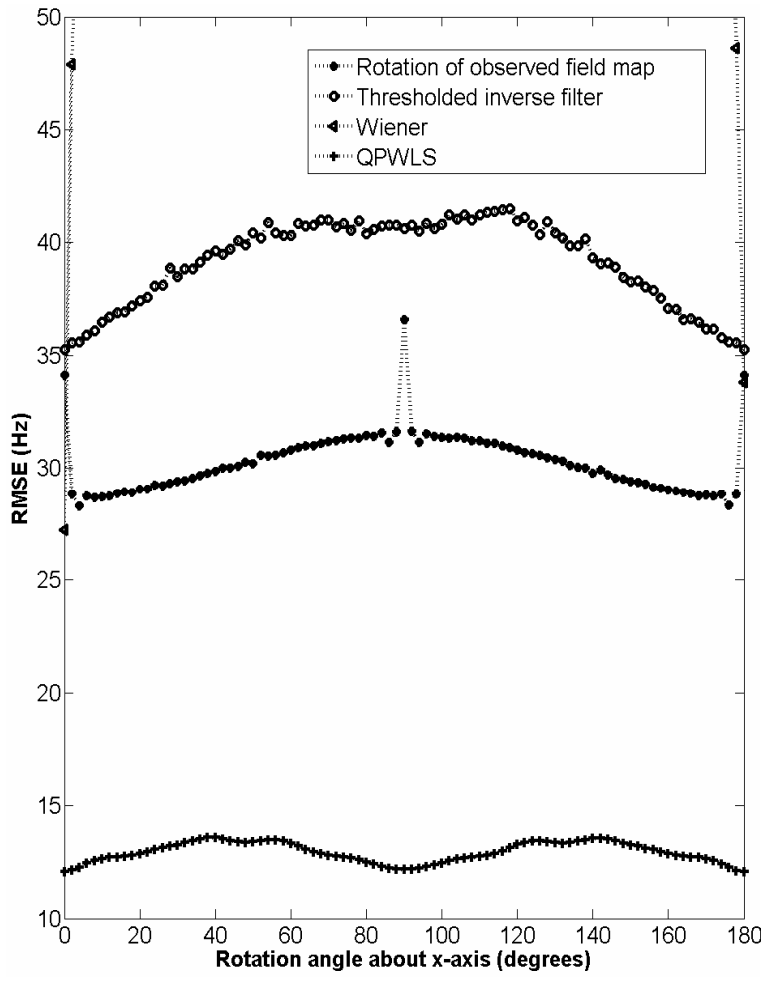

Fig. 2. Dynamic field map RMSE values versus rotation angles for different estimation methods when object was rotated about the $x$-axis from $0^{\circ}$ to $180^{\circ}$. The Wiener filter results were truncated due to large RMSEs. larization functions that utilize prior spatial information specific to a brain's $\chi$-map. A potential limitation of the proposed method may arise because $\Delta \boldsymbol{\omega}_{\text {sys }}$ was ignored in Eq. 11. In future work, we will investigate methods to reliably measure non- $\chi$ induced field inhomogeneities, characterize their effects on the various approaches in this work, and validate the proposed method with real data. 
A novel regularized image restoration approach to estimate field maps of a moving object was proposed and shown, with simulated data, to be more effective than nonregularized methods or simple transformations of an observed field map. In fMRI, this may potentially improve dynamic field map estimates and hence, geometric distortion correction accuracy.

\section{Acknowledgements}

This work was supported in part by the National Institute of Health grants 1P01 CA87634 and 8R01 EB00309.

\section{References}

1. Jezzard, P., Clare, S.: Sources of distortion in functional MRI data. Hum. Brain Mapp. 8, 80-85 (1999)

2. Cusack, R., Brett, M., Osswald, K.: An evaluation of the use of magnetic field maps to undistort echo-planar images. Neuroimage 18, 127-142 (2003)

3. Roopchansingh, V., Cox, R.W., Jesmanowicz, A., Ward, B.D., Hyde, J.S.: Single-shot magnetic field mapping embedded in echo-planar time-course imaging. Magn. Reson. Med. 50, 839-843 (2003)

4. Sutton, B.P., Noll, D.C., Fessler, J.A.: Dynamic field map estimation using a spiralin/spiral-out acquisition. Magn. Reson. Med. 51, 1194-1204 (2004)

5. Yeo, D.T.B., Fessler, J.A., Kim, B.: Concurrent correction of geometric distortion and motion using the map-slice-to-volume method in echo-planar imaging. Magn. Reson. Imag. 26, 703-714 (2008)

6. Truong, T.K., Clymer, B.D., Chakeres, D.W., Schmalbrock, P.: Three-dimensional numerical simulations of susceptibility-induced magnetic field inhomogeneities in the human head. Magn. Reson. Imag. 20, 759-770 (2002)

7. Yoder, D.A., Zhao, Y., Paschal, C.B., Fitzpatrick, J.M.: MRI simulator with objectspecific field map calculations. Magn. Reson. Imag. 22, 315-328 (2004)

8. de Munck, J.C., Bhagwandien, R., Muller, S.H., Verster, F.C., Van Herk, M.B.: The computation of MR image distortions caused by tissue susceptibility using the boundary element method. IEEE Trans. Med. Imag. 15, 620-627 (1996)

9. Schneider, E., Glover, G.: Rapid in vivo proton shimming. Magn. Reson. Med. 47, 335347 (1991)

10. Fessler, J.A., Yeo, D., Noll, D.C.: Regularized fieldmap estimation in MRI. In: Proc. IEEE Intl. Symp. Biomed. Imag., pp. 706-709 (2006)

11. Lim, J.S.: Two-dimensional signal and image processing. Prentice Hall, New Jersey (1990)

12. Fessler, J.A.: Image reconstruction toolbox (2007), http: //www. eecs.umich.edu/ fessler 